\title{
RESEARCH
}

Open Access

\section{Prefrontal fNIRS-based clinical data analysis of brain functions in individuals abusing different types of drugs}

\author{
Xuelin $\mathrm{Gu}^{1}$, Banghua Yang ${ }^{1 *}$, Shouwei Gao ${ }^{1 *}$, Lin Feng Yan², Ding Xu ${ }^{3}$ and Wen Wang ${ }^{2^{*}}$
}

\begin{abstract}
Background: The activation degree of the orbitofrontal cortex (OFC) functional area in drug abusers is directly related to the craving for drugs and the tolerance to punishment. Currently, among the clinical research on drug rehabilitation, there has been little analysis of the OFC activation in individuals abusing different types of drugs, including heroin, methamphetamine, and mixed drugs. Therefore, it becomes urgently necessary to clinically investigate the abuse of different drugs, so as to explore the effects of different types of drugs on the human brain.

Methods: Based on prefrontal high-density functional near-infrared spectroscopy (fNIRS), this research designs an experiment that includes resting and drug addiction induction. Hemoglobin concentrations of 30 drug users (10 on methamphetamine, 10 on heroin, and 10 on mixed drugs) were collected using fNIRS and analyzed by combining algorithm and statistics.
\end{abstract}

Results: Linear discriminant analysis (LDA), Support vector machine (SVM) and Machine-learning algorithm was implemented to classify different drug abusers. Oxygenated hemoglobin $(\mathrm{HbO} 2)$ activations in the OFC of different drug abusers were statistically analyzed, and the differences were confirmed. Innovative findings: in both the RightOFC and Left-OFC areas, methamphetamine abusers had the highest degree of OFC activation, followed by those abusing mixed drugs, and heroin abusers had the lowest. The same result was obtained when OFC activation was investigated without distinguishing the left and right hemispheres.

Conclusions: The findings confirmed the significant differences among different drug abusers and the patterns of OFC activations, providing a theoretical basis for personalized clinical treatment of drug rehabilitation in the future.

Keywords: Drug addiction, fNIRS, Clinical data analysis, Classification of different drug users, OFC activation

\footnotetext{
* Correspondence: yangbanghua@shu.edu.cn; swgao@shu.edu.cn;

wangwen@fmmu.edu.cn

${ }^{1}$ School of Mechanical and Electrical Engineering and Automation, Shanghai

University, Shanghai 200444, China

${ }^{2}$ Department of Radiology \& Functional and Molecular Imaging Key Lab of

Shaanxi Province, Tangdu Hospital, Fourth Military Medical University, Xi'an

710038, Shaanxi, China

Full list of author information is available at the end of the article
}

(c) The Author(s). 2021 Open Access This article is licensed under a Creative Commons Attribution 4.0 International License, which permits use, sharing, adaptation, distribution and reproduction in any medium or format, as long as you give appropriate credit to the original author(s) and the source, provide a link to the Creative Commons licence, and indicate if changes were made. The images or other third party material in this article are included in the article's Creative Commons licence, unless indicated otherwise in a credit line to the material. If material is not included in the article's Creative Commons licence and your intended use is not permitted by statutory regulation or exceeds the permitted use, you will need to obtain permission directly from the copyright holder. To view a copy of this licence, visit http://creativecommons.org/licenses/by/4.0/ The Creative Commons Public Domain Dedication waiver (http://creativecommons.org/publicdomain/zero/1.0/) applies to the data made available in this article, unless otherwise stated in a credit line to the data. 


\section{Background}

Clinically, there has been little theoretical support for the effects of brain functions abusing different types of drugs such as heroin, methamphetamine, and mixed drugs. Moreover, the high degree of OFC activations in drug users indicates a high degree of cravings for drugs and a high tolerance to punishment.

The paper mainly highlights the following six points: Forehead fNIRS is first applied to drug addiction clinics; Designing a special experimental paradigm process to collect clinical data of people taking different types of addictive drugs; Traditional evaluation of drug abuse types uses statistical scales and addicts' account, which is subjective. This article proposes using LDA, SVM and $\mathrm{CNN}$ algorithms to classify people taking different drugs, and objectively judge the types of drug abuse, overcoming the shortcoming of traditional evaluation mode of being subjective; Statistics is used to analyze the differences of OFC functional area activation; Warning people on social impacts, informing them of the harmfulness of drugs and necessity to refuse drugs; Clinically, providing a theoretical basis for doctors in differentiated rehabilitation treatment.

fNIRS is a new research field, and its advantages are received by researchers. Optical brain imaging functional near infrared spectroscopy $[1,2]$ is a spectral measurement based on scalp detection. It measures the hemodynamic function of brain tissues based on optic injection and detection points, and records blood oxygen level [3]. Compared with the emerging brain function imaging modality of EEG, NIRS is easy to wear, resistant to interference, and portable [4]. The oxyhemoglobin (HbO2) and deoxyhemoglobin ( $\mathrm{Hbb})$ in human body have specific absorption for the near-infrared light with a wavelength of $600 \mathrm{~nm}$ to $900 \mathrm{~nm}$, while other biological tissues in the brain are relatively transparent in this range of wavelength. Therefore, NIRS is an ideal choice to measure the changes in the intensity of nearinfrared light with a wavelength of $600 \mathrm{~nm}$ to $900 \mathrm{~nm}$ injected into the brain tissue, while the indirect brain function changes are measured with the hemodynamic data conversed based on the Beer-Lambertd law $[5,6]$. The near-infrared spectroscopy is widely used in evaluation, such as the evaluation of brain damage among drug users who take different types of drugs.

Dresler et al., using fNIRS, studied the neurotoxic effects of drinking and the nerve recovery related to alcohol withdrawal. They divided the experimental and control groups, designed the experiments, and obtained results compatible with an increase in frontal brain activity from alcohol dependence over abstinence up to normal functioning [7]. Okada, $\mathrm{N}$ et al. compared the activations in the prefrontal cortex between methamphetamine-associated psychosis and schizophrenia, and obtained the similarities and differences in prefrontal cortex dysfunction between the two conditions [8]. Yamamuro et al. Took Metaphetamine abuse as the research object. The Stroop Color word task experiment was designed, and the reduced hemodynamic responses in the prefrontal cortex might reflect higher levels of importance in patients with metaphase induced psychosis were obtained [9]. Ceceli et al. demonstrate the involvement of the prefrontal cortex in emotional, cognitive, and behavioral alterations in drug addiction, with particular attention to the impaired response inhibition and salience attribution (iRISA) framework. Consistent insights from human and non-human primate studies suggest that chronic drug use leads to iRISA-based prefrontal cortex damage [10].

Human prefrontal cortex (PFC) does not only participate in the generation and control of emotions, but is deemed to be closely related to attention, cognition and motivation $[11,12]$. fNIRS can detect changes in the activation of oxyhemoglobin and deoxyhemoglobin in the brain [13-16]. The NIRS detection results suggest abnormal activation of the prefrontal cortex, orbital frontal cortex and anterior cingulate gyrus among long-term methamphetamine users [17-21].

Relevant studies have found that there is a significant correlation between the neural activity in the orbitofrontal cortex and the behavioral indicators of rewards [22]. Findings on lesion studies show that orbitofrontal cortex plays a key role in cognitive flexibility [23]. Many studies on animals and humans have confirmed that damage to orbitofrontal cortex cost the cognitive flexibility of species [24, 25]. Existing studies on neuroimaging have confirmed the important role of human orbitofrontal cortex in backward learning. As orbitofrontal cortex is key to the regulation of cognitive and emotional processes (such as cognitive flexibility), regulation disorder would take place when neural changes occur in the orbitofrontal cortex [26-31]. The disorders further lead to addictive disorders. At the same time, given stimuli associated with negative results cannot change the decision-making behavior of addicts (who have high tolerance to punishment) [32, 33].

In this paper, different types of drug abusers were studied to explore the effects of abusing different drugs on the human body, so as to guide the clinical treatment for drug rehabilitation. Thirty drug abusers were selected based on the demographic scale, oral narration, and medical tests. Using the fNIRS device, the experimental paradigm was designed to obtain fNIRS data. First, the machine learning model was used to classify the different types of drug abusers. Then the statistical software was employed to analyze the OFC activations in individuals abusing different types of drugs. This study would be of great significance to the clinical treatment for drug rehabilitation. 


\section{Methods}

\section{Participates}

Study participate criteria: 1 . Meet the diagnostic criteria for disorders caused by DSM-5 psychoactive substances; 2. Patients within six months of the withdrawal period; 3. Junior high school education and above; 4. Years of Age 18-41; 5. Voluntary participation in this study and sign the informed consent form. Exclusion criteria: 1. Severe cognitive dysfunction, unable to cooperate with the completion of project-related assessment and testing; 2. Patients with severe physical diseases; 3 . Severe psychotic symptoms; 4 . Have other mental activities Substance abuse (except nicotine). The study was conducted in accordance with the declaration of Helsinki and was approved by the Ethics Committee of Shanghai University (Approval No. ECSHU2020-071).

\section{Drug users description}

According to the demographic scale made in the early stage, the most important items of 30 people (All male), such as drug use type, drug history, average drug dosage and drug use frequency, were accurately inquired and counted.

Methamphetamine easily causes intense excitement, which is difficult to eradicate after addiction.

Heroin is a psychoanaesthetic drug. Once a person becomes addicted, their physiological reaction is intense, and they have a compulsion to seek medication.

"Mixed drug abusers" refers to drug users who attempt to mix two or three drugs at a time. The main types of drugs used are: LSD, Flunitrazepam, $\mathrm{N}_{2} \mathrm{O}$, Pethidine hydrochloride, MDMA, Cannabis, ketamine, etc. Table 1 is the personal information of the selected 30 subjects.

\section{NIRS technology equipment introduction}

This paper uses a high-density NIRS device (NIRSIT; OBELAB, Seoul, Korea), and its specific hardware parameters and wearing methods are as follows: the specific hardware parameters are shown in Table 2. Figure 1 NIRSIT wearing method in the experiment.

Table 1 Personal information of drug addicts

\begin{tabular}{ll}
\hline $\mathbf{N}$ & $\mathbf{3 0}$ \\
\hline Sex(M/F) & Male \\
Age range (Year) & $19-41$ \\
Years of education & $10 \pm 2.72$ \\
Years of drug abuse & $8.2 \pm 4.74$ \\
Drug abuse per week: & Most people 3-5 times a week. \\
withdrawals & $1.4 \pm 0.98$ \\
Reasons for taking drugs & decompression needs ; sex ; \\
& emotion; curiosity; emotional \\
\end{tabular}

Table 2 NIRSIT specific hardware parameters

\begin{tabular}{ll}
\hline NIRSIT & OBELAB, Seoul, Korea \\
\hline Light source type & dual-wavelength VCSEL laser \\
Light source technology: & $\mathrm{CW}$ \\
Wavelength & $780 \mathrm{~nm}, 850 \mathrm{~nm}$ \\
Number of light sources & 24 \\
Number of detectors & 32 \\
SD distance & $1.5 \mathrm{~cm}, 2.12 \mathrm{~cm}, 3.0 \mathrm{~cm}, 3.35 \mathrm{~cm}$ \\
Number of channels & 204 \\
Detection distance & $0.2 \mathrm{~cm}, 0.6 \mathrm{~cm}, 1.0 \mathrm{~cm}, 1.4 \mathrm{~cm}, 1.8 \mathrm{~cm}$ \\
Spatial resolution & $4 \times 4 \mathrm{~mm}$ \\
Sampling frequency & $8.13 \mathrm{~Hz}$ \\
\hline
\end{tabular}

\section{NIRS channel and functional area division}

The four advanced functional areas detected by the forehead fNIRS device are divided into the dorsolateral prefrontal cortex, the ventrolateral prefrontal cortex, frontopolar prefrontal cortex, and the orbital frontal cortex. The specific channel distribution: the right dorsolateral prefrontal cortex is $1,2,3,5,6,11,17,18$ channels. There were 19, 20, 33, 34, 35, 38, 39 and 43 channels in the left dorsolateral prefrontal lobe. There are $4,9,10,40,44,45$ channels in ventrolateral prefrontal cortex of left and right hemispheres. There are 14, 15, 16, $29,30,31,32,46,47,48$ channels in the left and right orbital frontal cortex. Frontopolar prefrontal cortex is $7,8,12,13,21,22,23,24,25,26,27,28,36,37,41,42$ channels. Fig. 2 NIRS channel and functional area division.

\section{Near-infrared imaging theory}

When light passes through a uniform, non-scattering medium, only the absorption effect of the medium on the photons is considered. According to the Beer-Lambert law [34-37], the attenuation of light intensity is expressed as follows:

$$
O D=\log \frac{I}{I_{0}}=-\varepsilon(\lambda) c d \log \mathrm{e}
$$

where $I_{0}$ is the incident light intensity, $I$ is the incident light intensity, $\varepsilon(\lambda)$ is the extinction coefficient of the substance at a wavelength of $\lambda$, determined by the absorbing medium and the wavelength of the light, $c$ is the medium concentration, and $d$ is the thickness of the medium. The absorption coefficient $\mu_{a}$ is defined as follows:

$$
\mu_{a}=\varepsilon(\lambda) c
$$

The total absorption coefficient of the medium can be expressed as a linear superposition of the absorption coefficients of each medium: 


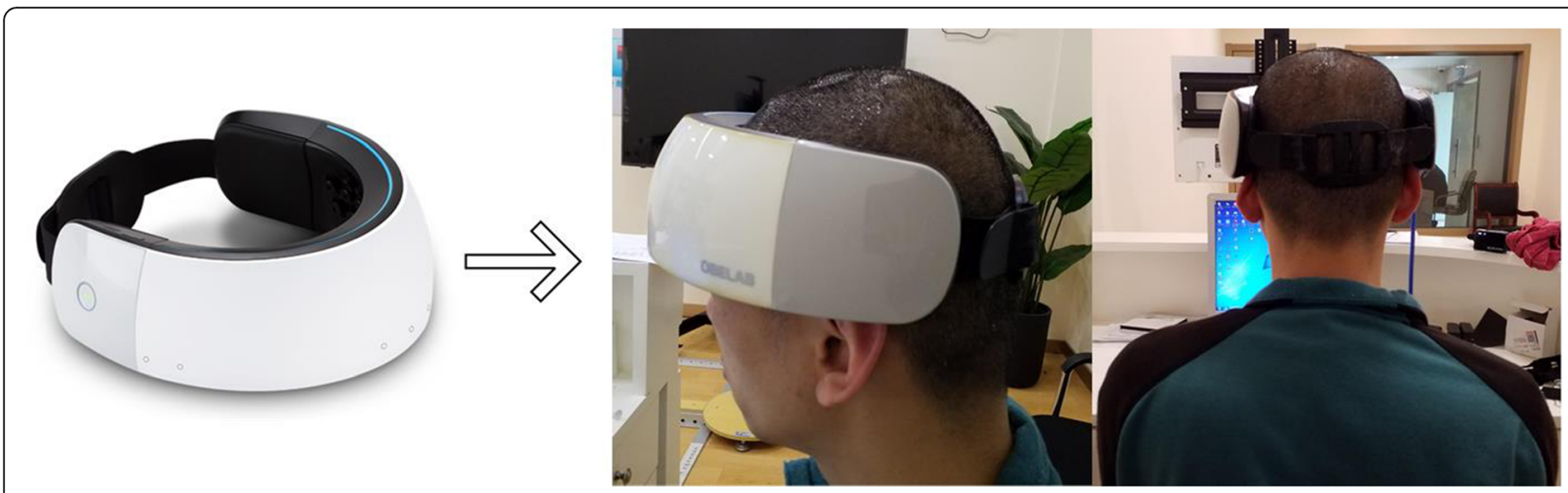

Fig. 1 NIRSIT wearing method in the experiment

$$
\mu_{a}(\lambda)=\sum_{i}^{N} \varepsilon_{i}(\lambda) c_{i}
$$

The optical density is the product of the medium thickness $d$ and the total absorption coefficient $\mu_{a}[38,39]$ :

$$
O D=\log \frac{I}{I_{0}}=d \sum_{i}^{N} \varepsilon_{i}(\lambda) c_{i}
$$

The actual biological tissue is very complex and is a strong light scatterer, and light undergoes multiple scatterings during its output before it can be detected. The attenuation of light in tissues includes absorption and scattering, and to capture the effect of scattering on light loss, Deply et al. proposed a modified Beer-Lambert law [40], expressed as follows:

$$
O D=\log \frac{I}{I_{0}}=D P F(\lambda) \cdot d c \varepsilon+G
$$

where $G$ denotes the light loss due to scattering and other boundary losses. DPF is the differential path factor, whose value is the ratio between the actual optical path length traveled by light in the tissue and $d$. The DPF values in different tissues can be obtained from the literature [41].

\section{Measurement of changes in hemodynamic parameters}

To detect changes in hemodynamic parameters using NIRS, a reference state is usually selected in nearinfrared measurements to detect relative changes in the concentration of absorbing chromophores $\Delta_{c}$, based on the modified Beer-Lambert law [42, 43]:

$$
\Delta O D=\log \frac{I}{I_{0}}=D P F(\lambda) \cdot d \Delta c \varepsilon
$$

When detecting relative changes in $\mathrm{HbO} 2$ and $\mathrm{HHb}$ concentrations:

$$
\Delta O D^{\lambda}=\left(\varepsilon_{H b o}^{\lambda} \Delta\left[H b O_{2}\right]+\varepsilon_{H H b}^{\lambda} \Delta[H H b]\right) D P F(\lambda) \cdot d
$$

where $\Delta\left[\mathrm{HbO}_{2}\right]$ and $\Delta[\mathrm{HHb}]$ are the variations in $\mathrm{HbO}_{2}$ and $\mathrm{HHb}$ concentrations, the selected incident near-infrared wavelengths $\lambda_{1}$ and $\lambda_{2}$, brought into the above equation have $[44,45]$.

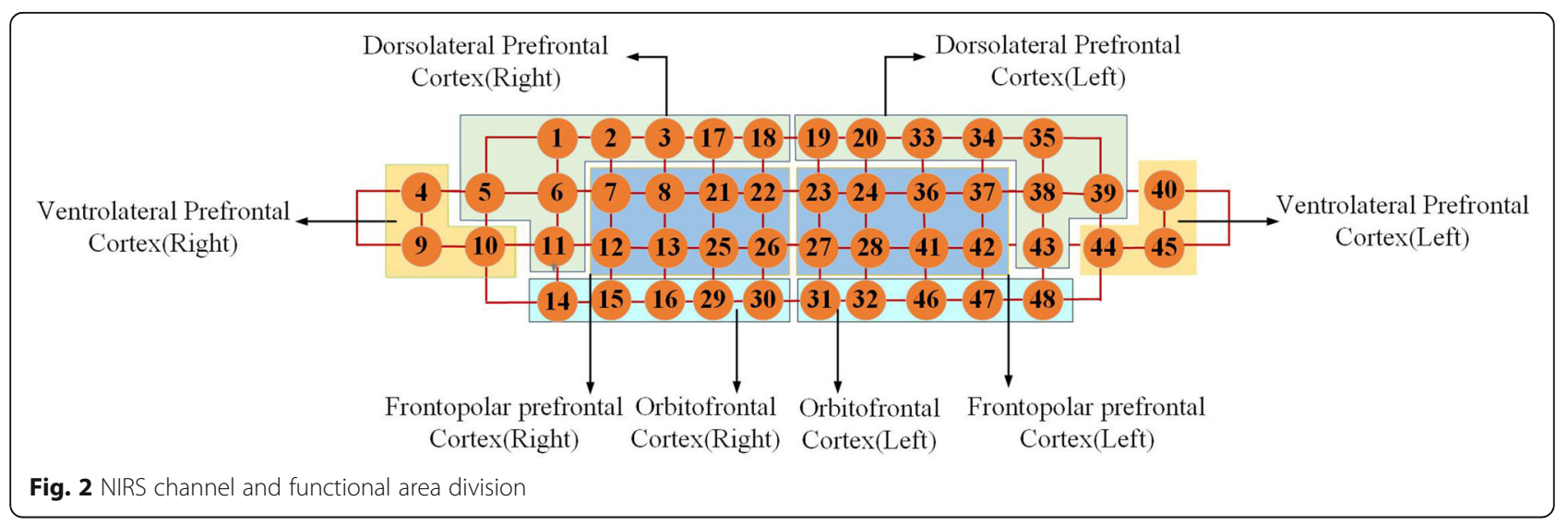




$$
\begin{aligned}
& \Delta O D^{\lambda_{1}}=\left(\varepsilon_{H b o}^{\lambda_{1}} \Delta\left[H b O_{2}\right]+\varepsilon_{H H b}^{\lambda_{1}} \Delta[H H b]\right) D P F\left(\lambda_{1}\right) \cdot d \\
& \Delta O D^{\lambda_{2}}=\left(\varepsilon_{H b o}^{\lambda_{2}} \Delta\left[H b O_{2}\right]+\varepsilon_{H H b}^{\lambda_{2}} \Delta[H H b]\right) D P F\left(\lambda_{2}\right) \cdot d
\end{aligned}
$$

When the value of DPF is known, solving the above equation for the system of equations yields the change in $\mathrm{HbO}_{2}$ concentration $\triangle\left[\mathrm{HbO}_{2}\right]$ and the change in $\mathrm{HHb}$ concentration $\triangle[H H b]$, expressed as follows:

$$
\begin{gathered}
\Delta\left[H b O_{2}\right]=\frac{\varepsilon_{H H b}^{\lambda_{2}} \frac{\Delta O D^{\lambda_{1}}}{D P F\left(\lambda_{1}\right)}-\varepsilon_{H H b}^{\lambda_{1}} \frac{\Delta O D^{\lambda_{2}}}{D P F\left(\lambda_{2}\right)}}{d\left(\varepsilon_{H H b}^{\lambda_{2}} \varepsilon_{H b O_{2}}^{\lambda_{1}}-\varepsilon_{H H b}^{\lambda_{1}} \varepsilon_{H b O_{2}}^{\lambda_{2}}\right)} \\
\Delta[H H b]=\frac{\varepsilon_{H b O_{2}}^{\lambda_{2}} \frac{\Delta O D^{\lambda_{1}}}{D P F\left(\lambda_{1}\right)}-\varepsilon_{H b O_{2}}^{\lambda_{1}} \frac{\Delta O D^{\lambda_{2}}}{D P F\left(\lambda_{2}\right)}}{d\left(\varepsilon_{H H b}^{\lambda_{2}} \varepsilon_{H b O_{2}}^{\lambda_{1}}-\varepsilon_{H H b}^{\lambda_{1}} \varepsilon_{H b O_{2}}^{\lambda_{2}}\right)}
\end{gathered}
$$

\section{Experiment design and data acquisition}

We used E-prime software package (Psychology Software Tools, Pittsburgh, PA) to write the experimental paradigm, with each map numbered. A complete experimental paradigm consists of the following three stages.

The first stage of the experimental paradigm, $10 \mathrm{~min}$ in total, during which the subjects need to close their eyes for $5 \mathrm{~min}$ and then open their eyes for $5 \mathrm{~min}$.

The second stage, it lasts $6 \mathrm{~min}$ and is divided into drug maps and neutral maps. Among them, each block lasts $10 \mathrm{~s}$. There are a total of 16 maps, and the display time of each map is $0.6 \mathrm{~s}$. At the beginning, the first four maps are displayed randomly, during which there are two drug maps. After displaying the first four maps, the remaining 12 neutral maps are displayed randomly. After a block ends, there will be a 4-s interval map with a white background and a black cross. Figure 3, the examples of drug abuse-related maps used in the experimental paradigm.

The third stage, it lasts a total of $4.6 \mathrm{~min}$, during which the maps are all neutral, with each block lasting $10 \mathrm{~s}$. There are 16 maps in total, with a display speed of $0.6 \mathrm{~s}$. There will be a 4 -s interval between each block. Figure 4, the examples of neutral maps used in the experimental paradigm. Figure 5, The whole process of experimental paradigm.

\section{Linear discriminant analysis algorithm principle}

Linear discriminant analysis (LDA) is a supervised pattern recognition method. The LDA classifier reduces the dimensions of the data, reduces complex features into low-dimensional features through projection, and searches for specific classification surfaces to maximize the discrimination between the two types of task classification in order to realize feature classification [46].

LDA is the most traditional linear classifier; there are $\mathrm{k}$ linear functions for a $\mathrm{k}$-classification problem.

$$
y_{K}(x)=w_{k}^{T} x+w_{k_{0}}
$$

When $y_{k}>y_{j}$ for all $\mathrm{j}$, then $\mathrm{x}$ belongs to class $\mathrm{k}$. When $k=2$, it becomes a binary problem.
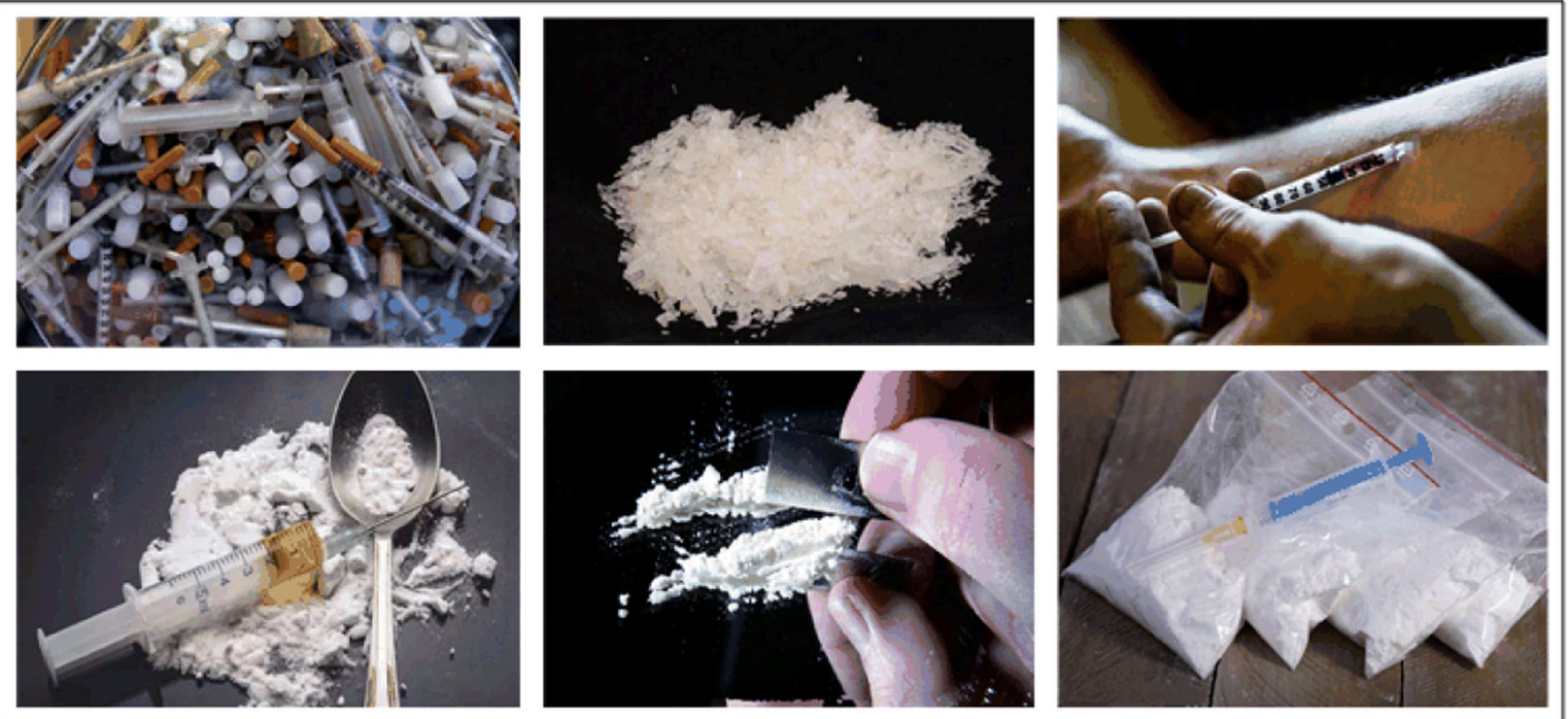

Fig. 3 Examples of drug abuse-related maps in the experimental paradigm 


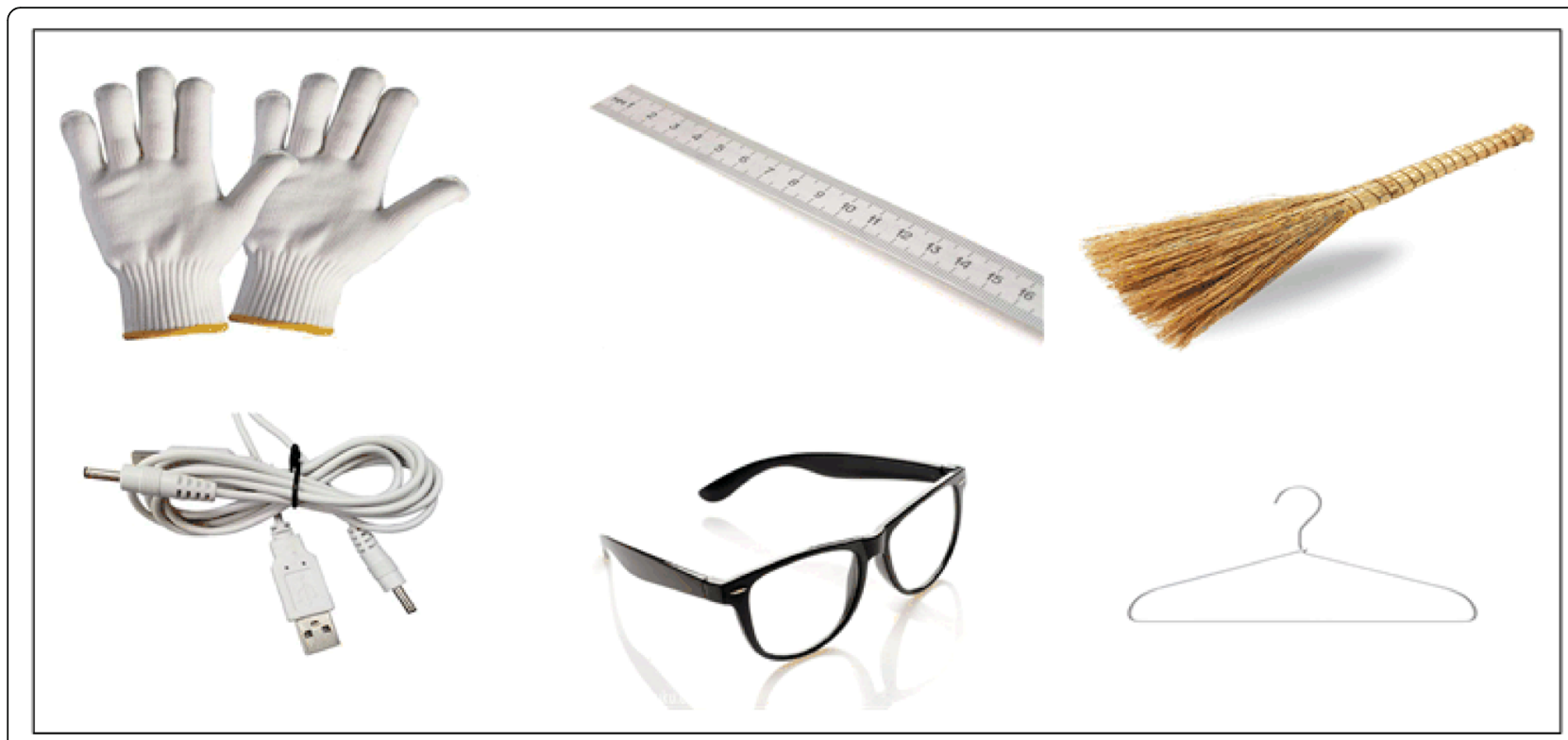

Fig. 4 Examples of neutral maps in the experimental paradigm

\section{Support vector machine algorithm principle}

Support vector machine (SVM): For nonlinear feature input, we design mapping rules to map nonlinear features to highdimensional space and ensure that the features are distributed linearly in high-dimensional space. Then, we can easily construct the optimal classification hyperplane in highdimensional space based on the structural risk minimization criterion so that this optimal classification hyperplane can correctly separate as many of the two types of samples on the one hand and maximize the classification interval between the two types on the other [47].

The sample set is designated as $\left(x_{i}, y_{i}\right), i=1,2, \cdots, l, x_{i} \in R^{d}$, where $y_{i} \in\{1,-1\}$ is the category number. The linear separable and linear non-separable cases are explored jointly, and the relaxation variable is introduced $\xi_{i} \geq 0$, where $\xi_{i}=0$ represents linear separability, and $\xi_{i}>0$ represents nonlinear separability. If the classification surface equation is $w \cdot x+b=0(w$ is the weight vector, and $b$ is the offset), the classification interval is equal to $\frac{2}{\|w\|}$. Maximizing the classification interval is equivalent to minimizing $\|w\|$ or $\left(\|w\|^{2}\right)$. To make the classification surface classify all samples correctly as much as possible, it is necessary to meet the following constraints:

$$
y_{i}\left[\left(w \cdot x_{i}\right)+b\right]-1+\xi_{i} \geq 0, i=1,2, \cdots, l
$$

Therefore, if the constraint formula is satisfied, the $\left(\|w\|^{2}\right)$ minimum classification surface can be the optimal classification surface.

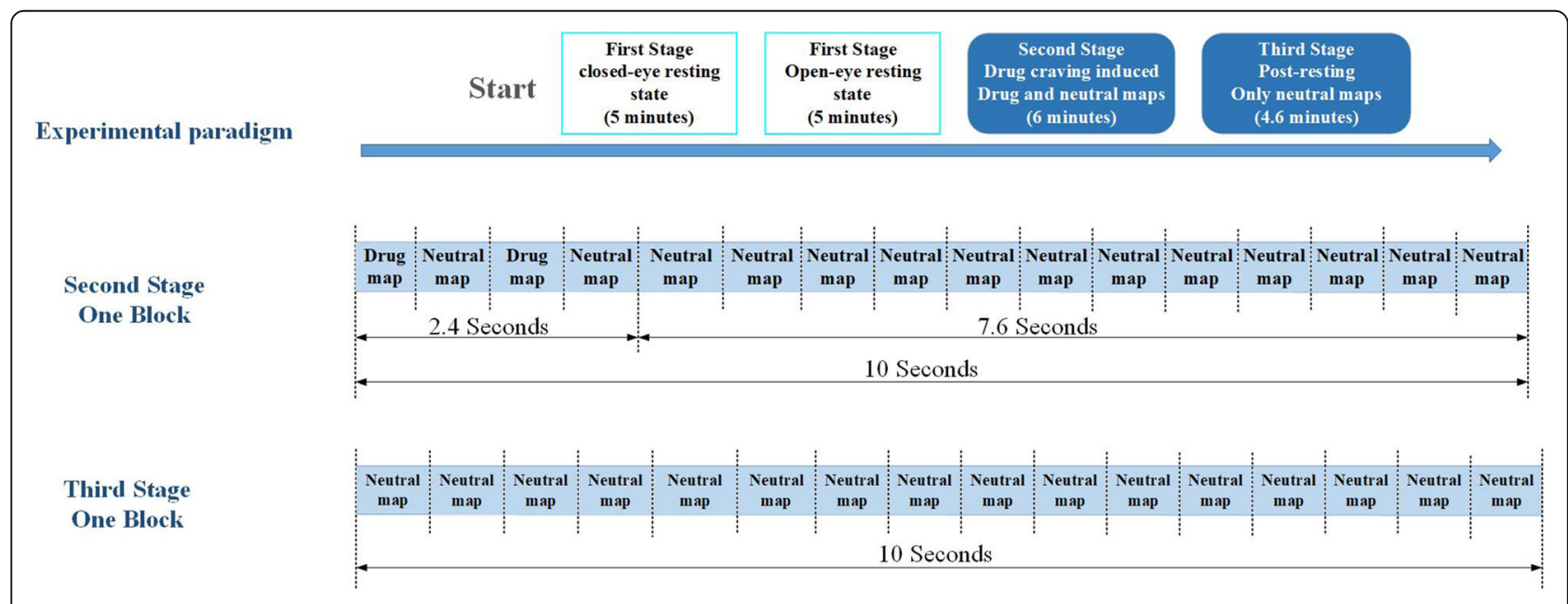

Fig. 5 The whole process of experimental paradigm 


\section{Machine learning algorithm classification}

In this paper, we propose and design a neural network algorithm model that extracts the hidden features in the 0.625 -s fNIRS data after drug map stimulation by convolution to define the type of drugs of abuse. In this paper, a convolutional neural network (CNN) model is designed. It is used to implement the classification of people who abuse different type of addictive drugs. The Structure of CNN used in this paper is shown in Fig. 6.

\section{Data preprocessing}

Butterworth filter, the frequency response curve in the passband has a relatively flat and undulating character, and gradually decreases to zero at the edge of the stopband. In this paper, a Butterworth filter based on infinite impulse response is selected to band-pass filter the acquired Near infrared signals for the purpose of physiological artifact removal. The expression for the n-order Butterworth filter is as follows:

$$
|H(f)|^{2}=\frac{1}{1+\left(\frac{f}{f_{c}}\right)^{2 n}}=\frac{1}{1+\mathbf{\epsilon}^{2}\left(\frac{f}{f_{p}}\right)^{2 n}}
$$

Where $\mathrm{n}$ is the order, $\boldsymbol{f}_{\boldsymbol{c}}$ is the cutoff frequency, and $\boldsymbol{f}_{\boldsymbol{p}}$ is the passband edge frequency.

In this paper, $\mathrm{n}$ is 6 and the frequency band range is $0.01 \mathrm{~Hz}$ to $3 \mathrm{~Hz}$. This band range can remove the interference of heartbeat respiration and slow drift to the raw data, and also can maximize the preservation of hemodynamic characteristics.

The formula for each structure in the CNN network is as follows:

\section{Convolutional layer}

Convolutional layers are the core of convolutional neural networks [48]. The calculation form is as follows:

$$
x_{j}^{l}=f\left(\sum_{i \in M_{j}} x_{i}^{l-1} \cdot k_{i j}^{l}+b_{j}^{l}\right)
$$

$x_{j}^{l}$ is the $j$ th feature of the layer $l . k_{i j}^{l}$ is the $j$ th feature of the layer $l$ and the $i$ th feature of the layer $l-1 . b_{j}^{l}$ is a bias parameter, $f(\bullet)$ is the activation function.

\section{Pooling layer}

The pooling layer sub-samples the input features according to specific rules in order to make the network robust to small changes in previously learned features [49]. The calculation form is as follows:

$$
x_{j}^{l}=f\left(\beta_{1}^{l} \backslash \operatorname{down}\left(x_{j}^{l-1}\right)+b_{j}^{l}\right)
$$

$x_{j}^{l}$ is the $j$ th feature of the layer $l . \beta_{1}^{l}$ is the Subsampling coefficient. $b_{j}^{l}$ is the bias parameter, $\operatorname{down}(\bullet)$ is a subsampling function, $f(\bullet)$ is the activation function.

\section{Normalization of data}

Batch standardization layer: in training convolutional neural network, the input data are usually whitened, which can speed up the training speed.

Set the data value input $=\left\{x_{1} \ldots x_{m}\right\}$ of the input data block, the parameters to be learned are $\gamma$ and $\beta$, first calculate the average value of each data block:

$$
\mu_{B}=\frac{1}{m} \sum_{i=1}^{m} x_{i}
$$

Calculate the variance of each data block:

$$
\sigma_{B}^{2}=\frac{1}{m} \sum_{i=1}^{m}\left(x_{i}-\mu_{B}\right)^{2}
$$

Normalize each set of data:

$$
\hat{x}_{i}=\frac{x_{i}-\mu_{B}}{\sqrt{\sigma_{B}^{2}+\varepsilon}}
$$

Using the parameters that need to be learned in the network and linear transformation:

$$
y_{i}=\gamma \hat{x}_{i}+\beta \equiv B N_{\gamma \beta}\left(x_{i}\right)
$$

\section{Activation function}

In this paper, the activation function uses a modified linear unit (ReLU), and the corresponding calculation formula is as follows:

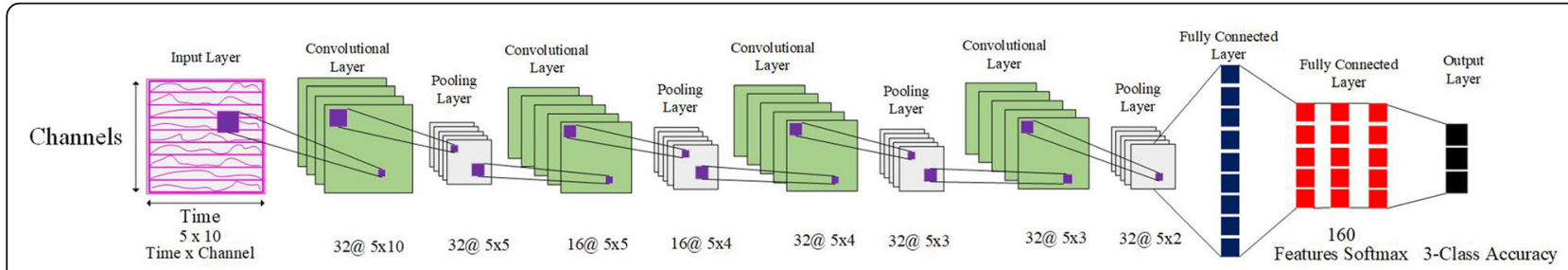

Fig. 6 Structure of convolutional neural network 


$$
\operatorname{ReLU}=\left\{\begin{array}{c}
x, x \geq 0 \\
x, x<0
\end{array}=\max (0, x) .\right.
$$

The results show that the derivation of the activation function is simple, and the output of some neurons is 0 , which realizes the sparsity of the network, reduces the interdependence of parameters [50].

\section{Full connection layer}

Each feature must be converted to one-dimensional before it can be used as the input of the fully connected layer [51]. The calculation as follows:

$$
h_{w, b}(x)=\theta\left(w^{\mathrm{T}} x+b\right)
$$

$h_{w, b}(x)$ is the output value of the neuron. $x$ is the input feature vector of the neuron. $w$ is the weight. $b$ is the bias parameter. $\theta(\cdot)$ is the activation function; The first fully connected layer in this paper uses the ReLU activation function.

\section{Softmax layer}

In $\mathrm{CNN}$, if the final output result is single-label multiclassification, the softmax function is usually used to normalize and map to the probability value, and the Softmax calculation formula is as follows:

$$
z_{i}=\operatorname{Softmax}\left(o_{i}\right)=\frac{\exp \left(o_{i}\right)}{\sum_{c} \exp \left(o_{c}\right)}
$$

$o_{i}$ is the value of the output neuron corresponding to the Ith category.

\section{Results}

\section{Accuracy comparison of LDA, SVM, and CNN}

LDA and SVM use the original data as the training set and test set. The classification model selects 21 data points as the training set and 9 as the test set and cross validates the model. Finally, we obtain their own 3-class accuracy. The classification accuracy of LDA is stable between 45 and $58 \%$. The classification accuracy of SVM is stable between 60 and $69 \%$.

The feature extraction method and classification accuracy of the CNN model are as follows:

The data feature 16 channels and 56 trials for each subject. The data fragment is $0.625 \mathrm{~s}$ after the drug picture appears. The CNN network includes 24 subjects' training data, 3 subjects' validation data and 3 subjects' testing data.

The CNN model shows a stable decreasing trend in loss and convergence after 1200 epochs, which proves that the network structure is stable. After several experiments, the optimal model basically appears between 1000 and 1300 epochs. At this time, the 3-class accuracy of different drug abusers is between 70 and $80 \%$. The accuracy rates tested using LDA, SVM, and CNN algorithms, respectively, are given in the table. A comparison between the three is shown in the figure. Table 3 Threeclass accuracy statistics. Figure 7 Comparison of 3-class accuracy between LDA, SVM and CNN.

\section{Analysis methods of drug abusers}

Statistical analyses were performed using GraphPad Prism 6.5 software (GraphPad Software Inc., San Diego, USA). The independent sample $t$-test and the nonparametric Mann-Whitney $U$ test were used in the study. The relevant laws of brain activation of people who abuse different drugs were obtained. Especially in the OFC functional area of the brain, people who abuse different drugs obtain significantly different results.

Right-OFC activated for people who abuse different drugs To begin with, five channels in both the left and right hemispheres of the brain of 30 addicts who take heroin, methamphetamine and mixed drugs were chosen to get the near-infrared data induced by drug pictures. Then the mean of OFC data of 30 subjects was calculated. The data of each type of drug addicts was saved in one column and processed with GraphPad Prism6.5 software.

For Heroin VS Methamphetamine in right OFC, the independent sample $\mathrm{T}$ test results were $\mathrm{t}=1.020, \mathrm{df}=98$, $P=0.312, \mathrm{~F}=31.90, \mathrm{DFn}=49, \mathrm{Dfd}=49, P<0.0001$; and for Mixed drug VS Methamphetamine, the results were $\mathrm{t}=0.7409, \mathrm{df}=98, P=0.4605, \mathrm{~F}=1.048, \mathrm{DFn}=49, \mathrm{Dfd}=$ $49, P=0.8707$.

For Heroin VS Mixed drug, the results were $\mathrm{t}=0.8400$, $\mathrm{df}=98, P=0.4030, \mathrm{~F}=33.42, \mathrm{DFn}=49, \mathrm{Dfd}=49, \quad P<$ 0.0001 . This is caused by heterogeneity variance. For Heroin VS Methamphetamine, the results of the nonparametric Mann Whitney test were Exact, Two-tailed, $P=0.0048<0.05$ (significant difference); for Methamphetamine VS Mixed drug, the results were Exact, Twotailed, $P=0.2215$ (insignificant difference); and for Her-

\begin{tabular}{|c|c|c|c|c|c|c|c|c|c|c|}
\hline & 1 & 2 & 3 & 4 & 5 & 6 & 7 & 8 & 9 & 10 \\
\hline LDA & $58.24 \%$ & $54.29 \%$ & $53.83 \%$ & $45.66 \%$ & $49.52 \%$ & $48.90 \%$ & $47.96 \%$ & $46.19 \%$ & $45.24 \%$ & $45.91 \%$ \\
\hline SVM & $69.39 \%$ & $68.57 \%$ & $66.90 \%$ & $64.83 \%$ & $64.29 \%$ & $63.27 \%$ & $62.38 \%$ & $62.09 \%$ & $61.81 \%$ & $60 \%$ \\
\hline CNN & $77.04 \%$ & $72.96 \%$ & 74.74\% & 75.77\% & $75.26 \%$ & $74.59 \%$ & 73.47\% & $76.79 \%$ & 73.47\% & $76.28 \%$ \\
\hline
\end{tabular}
oin VS Mixed drug, the results were Exact, Two-tailed,

Table 3 3-class accuracy statistics 


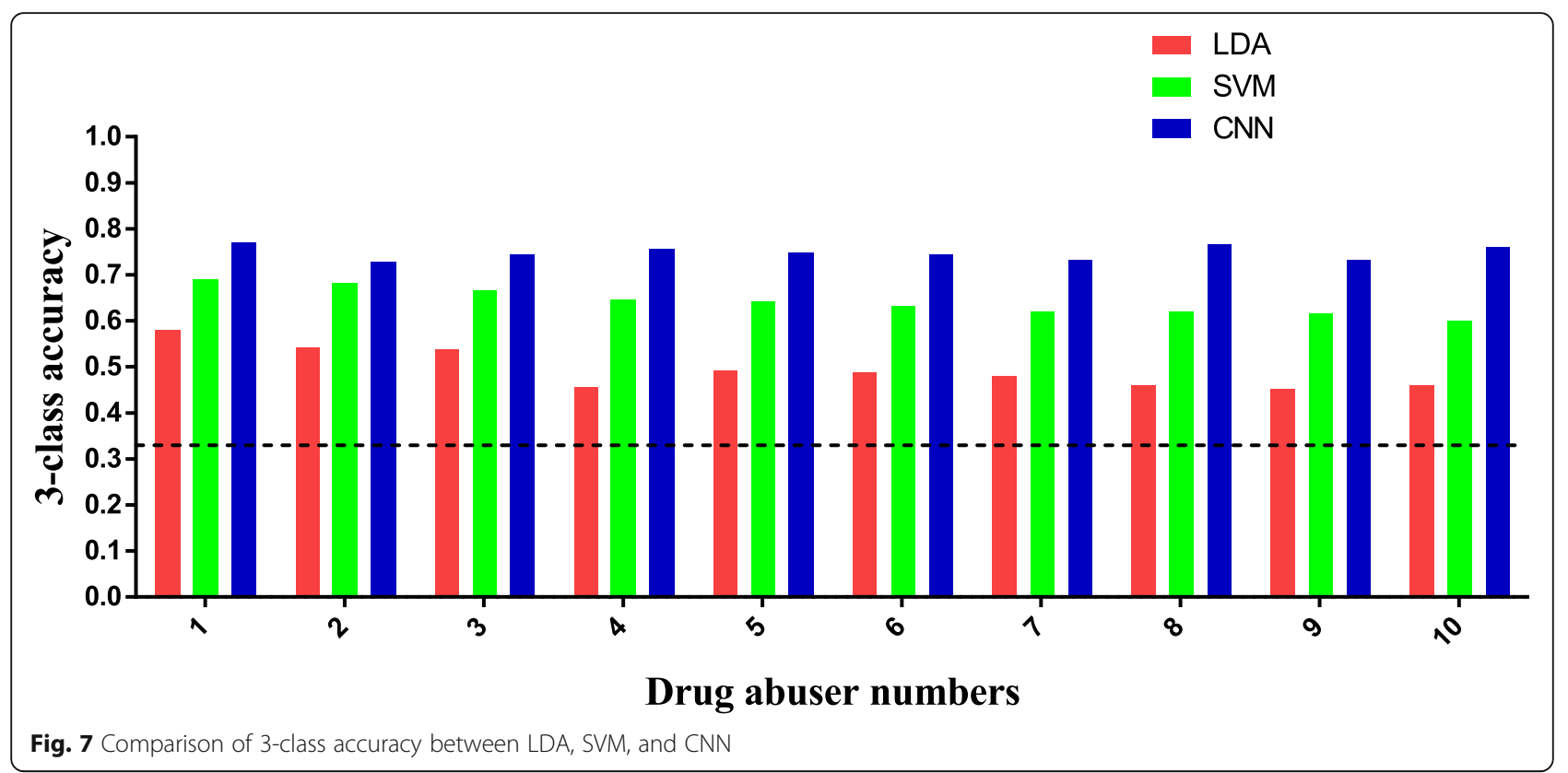

$P=0.0852$ (insignificant difference). As for the activation of right OFC by three types of drugs (in $\mathrm{mMol} / \mathrm{L}$ ), the $\mathrm{HbO} 2$ of heroin addicts was $-0.0007433 \pm 0.04683$, methamphetamine addicts $0.006117 \pm 0.008292$, and mixed addicts $0.004902 \pm 0.00810$. The activation of right OFC is highest among methamphetamine addicts, followed by mixed types and heroin. The result is the same for left- OFC. Figure 8, Right-OFC and Left-OFC activation differences among the three types of drug abusers.

\section{Activation of the left and right hemispheres of the brain} in drug addicts

For methamphetamine, mixed drugs, and heroin addicts, the left and right hemispheres of the brain are activated in OFC. The average values of the oxygenated hemoglobin concentration and deoxygenated hemoglobin concentration of the three types of subjects within $0-10 \mathrm{~s}$ are shown in Fig. 9. There is a difference in activation between the left and right hemispheres of the brain.

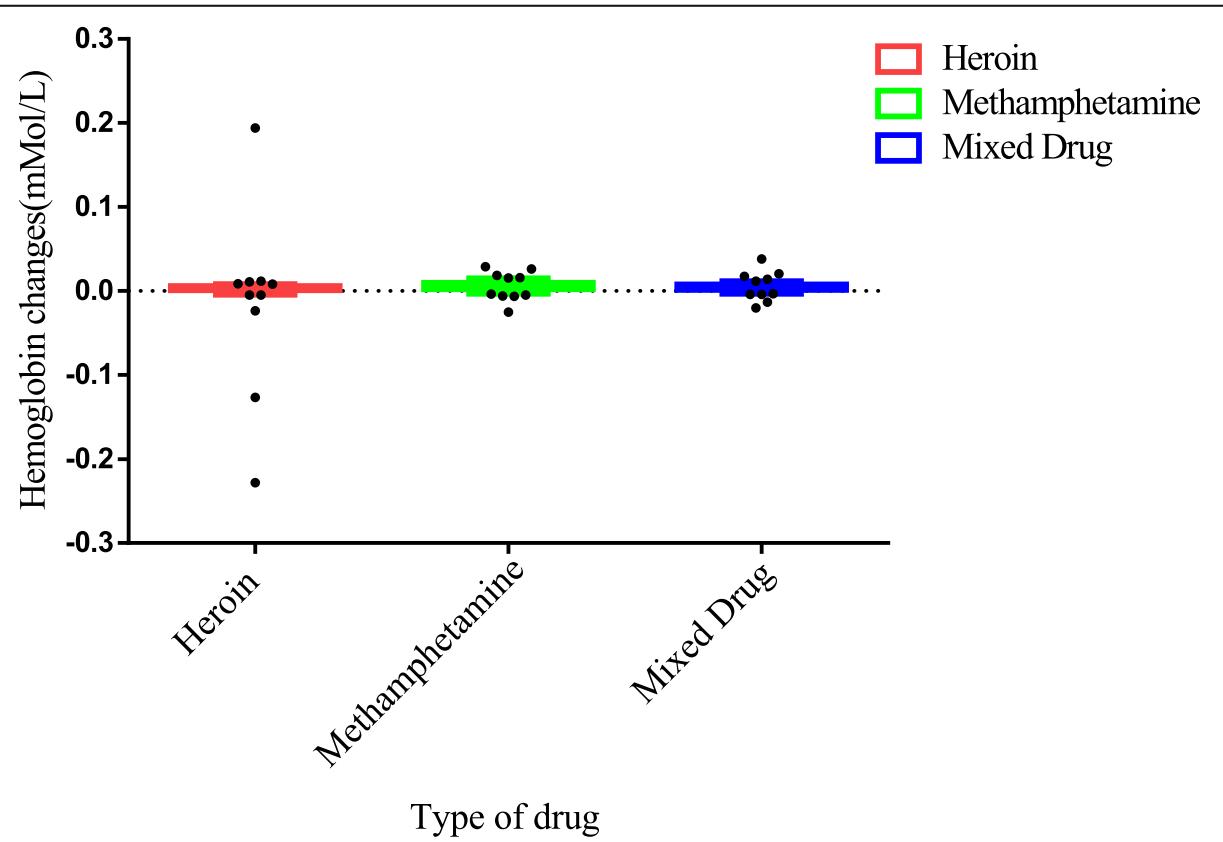

Fig. 8 Right-OFC and Left-OFC activation differences among the three types of drug abusers 
Methamphetamine-Task1-Subject Average
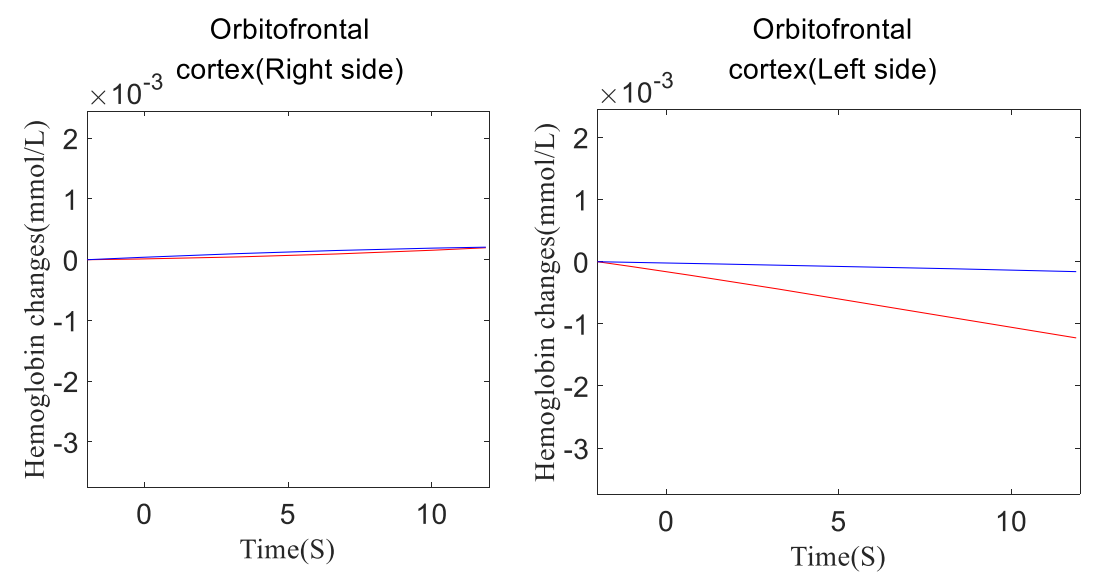

Mixed drug-Task1-Subject Average

Orbitofrontal cortex(Right side)

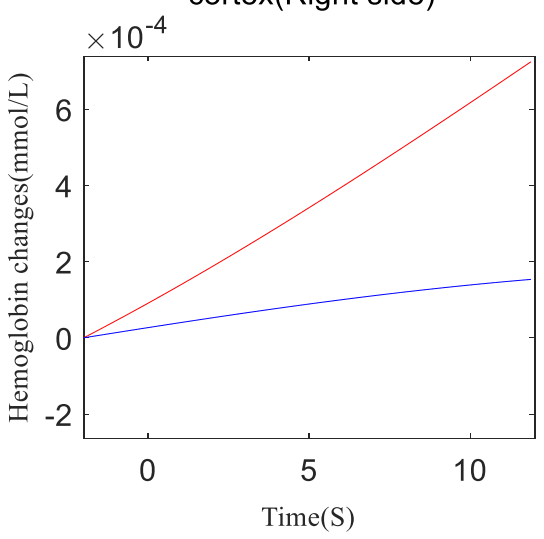

Orbitofrontal cortex(Left side)

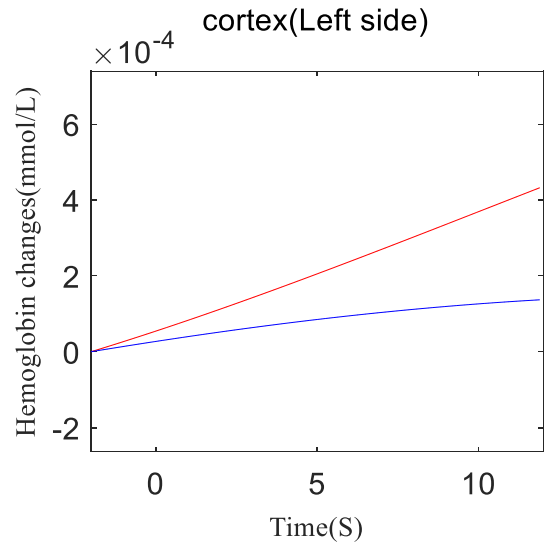

Heroin-Task1-Subject Average

Orbitofrontal

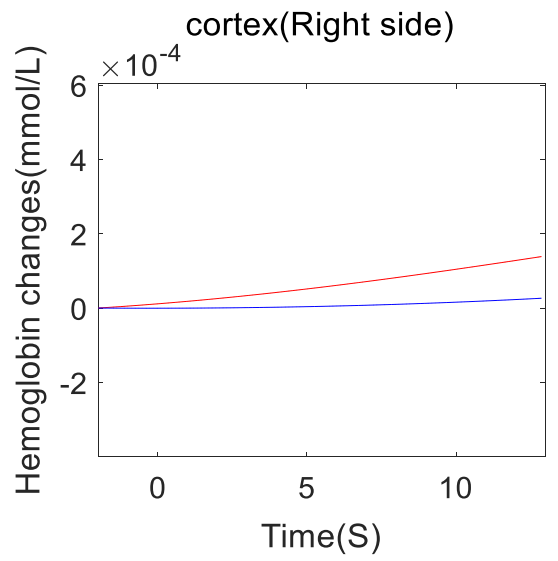

Fig. 9 time series of hemoglobin concentration

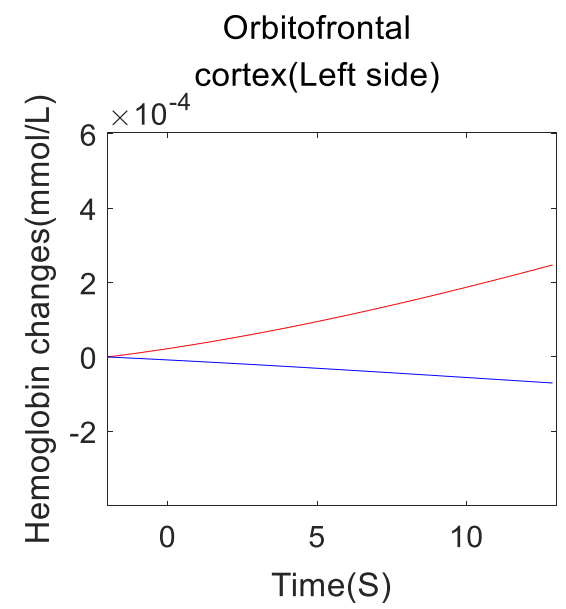




\section{Abuse of different drugs differs in OFC activation} The results of activation of the three types of personnel on OFC were analyzed using a non-parametric KruskalWallis test. The number of tested groups was three, and the total number of values was 300 . The Kruskal-Wallis test statistic was $8.355(P=0.0153)$. There were significant differences among the three types of personnel. Activation of the right OFC was the highest among methamphetamine addicts, followed by mixed types, and lastly, heroin addicts. Figure 10 shows differences in OFC activation among the three types of drug abusers.

\section{Differences in prefrontal cortex activation across drug addicts}

From the prefrontal cortex activation results in Fig. 11, we can see the differences in activation of the OFC cortex in the black box. The darker the red color, the higher the degree of activation. Methamphetamine abusers had the highest average blood oxygen levels in the OFC, followed by mixed drug abusers, and heroin abusers had the lowest.

\section{Discussion}

This study first uses the LDA, SVM and machinelearning algorithm to classify the addicts of heroin, methamphetamine and mixed drugs. Analysis on the brain activation and OFC activation is made to three types of drug addicts, which is of clinical significance.
Based on the analysis of addicts who take different types of drugs, the research finds that the activation of right OFC is highest among methamphetamine addicts, followed by mixed types and heroin. The result is the same for left OFC, but with significant difference in the methamphetamine group. The findings show the difference in the impact on human bodies among addicts who take heroin, methamphetamine and mixed drugs.

The final results obtained demonstrate that the abuse of different drugs produces differences in OFC activation. OFC activation changes after the addicts use drugs. The results are consistent with those arrived at by other scholars. Some researchers only proved the difference in OFC between drug addicts and healthy people. No research has been made to study the differences in prefrontal cortex activation caused by different drugs.

The four characteristics of addiction: impaired cognitive and motivational functions, increased craving for addictive cues, preference for rewards while ignoring risks, and impulse control disorder [52-54]. Another typical feature of addiction is that the individuals tend to pursue rewards for addictive behaviors while ignoring the risks in their cognitive decision-making $[55,56]$. Heroin addicts showed higher impulsivity in the Go/Nogo experimental tasks, and the activation in their cognitive control loop was weakened [57]. Long-term abuse of addictive drugs not only harms the physical and mental health of addicts, but also impairs their social functions.

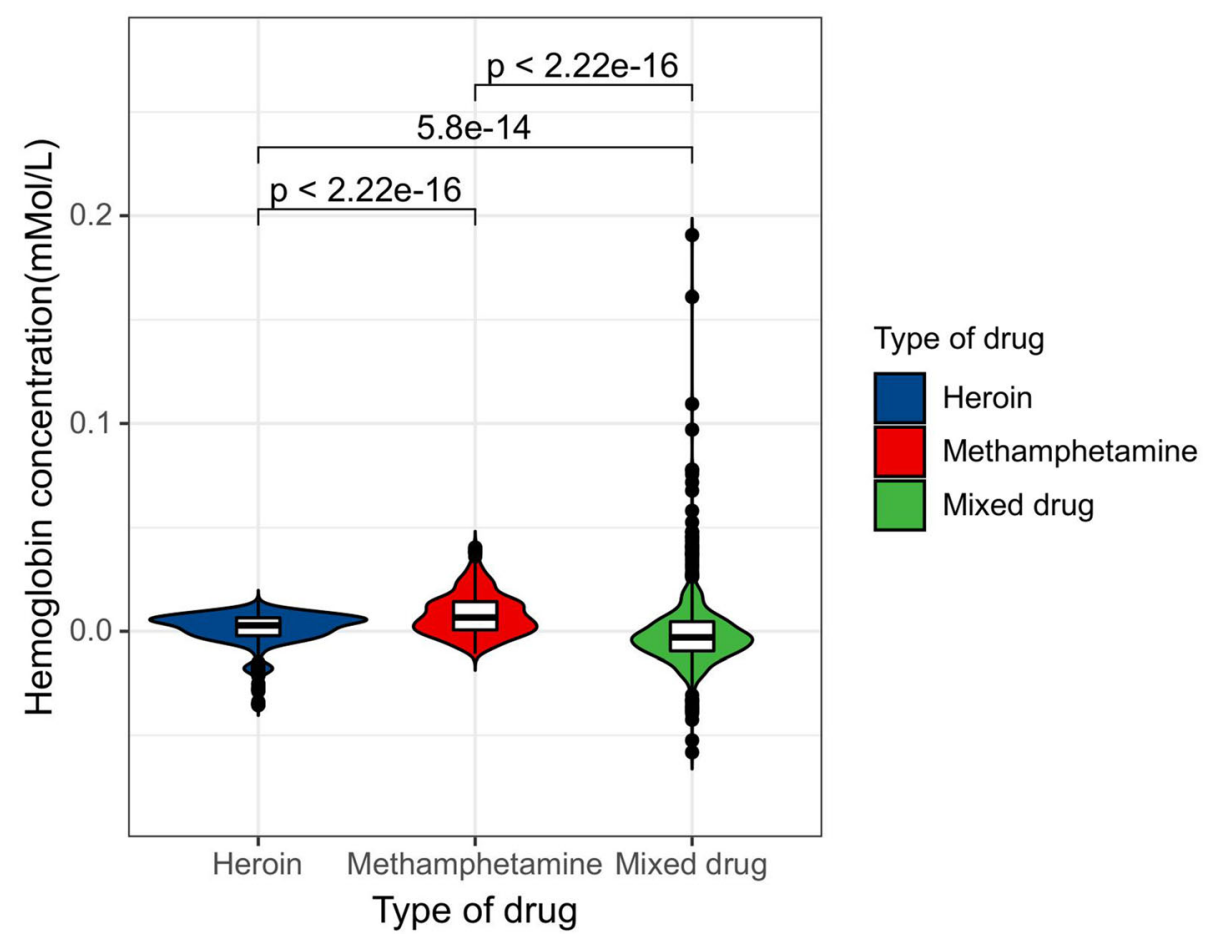

Fig. 10 OFC activation differences among the three types of drug abusers 


\section{Methamphetamine}

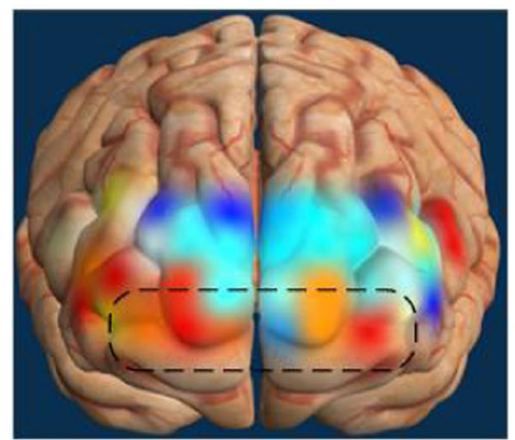

Mixed drug

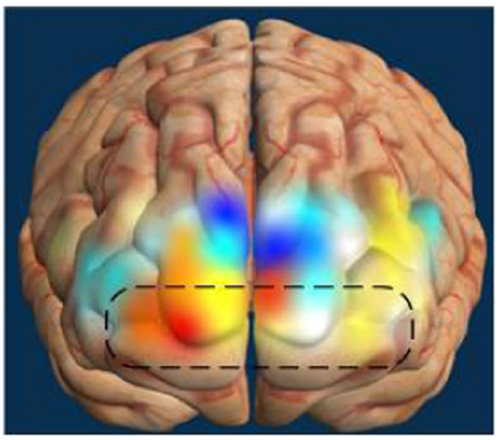

Heroin

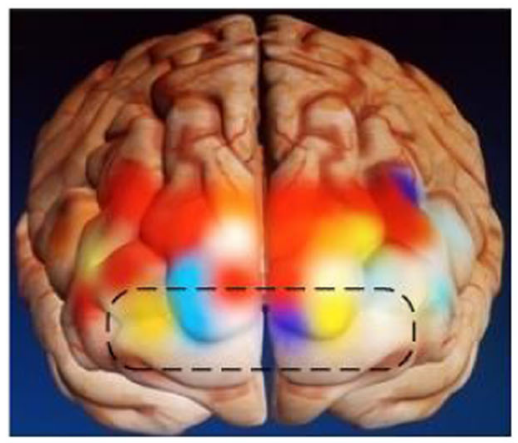

Fig. 11 Taking different type of drug addicts brain OFC activation

Meanwhile, it also causes serious damages to the cognitive processes of addicts, such as focus and concentration, verbal memory, and executive capacity $[58,59]$. At present, a large amount of evidence shows that the brain areas related to the cognitive and emotional processing of drug addicts are damaged to varying degrees by the abuse of addictive drugs [60].

Changes in OFC activation cause changes in cognition, control, and emotions. Differences in OFC activation among addicts who take different types of drugs lead to the conclusions that: Methamphetamine has the most serious impact on the human body; There are differences in the harm of different drugs; Customized drug rehabilitation schemes should be developed; Cognitive and control training is conducive to drug rehabilitation.

The limitation of this research lies in the limited amounts of subjects, which could make the findings less convincing. In future experiments, more subjects should be included and comparison between the outcomes of customized rehabilitation and those of traditional rehabilitation should be made.

The experiment proves that different drugs have varied impacts on the addicts, which requires customized drug rehabilitation schemes.

\section{Conclusion}

This paper points out that different drugs affect the brain differently. The brain activation pattern of heroin, methamphetamine and mixed drugs is arrived at, filling up the blank on the study about different types of drugs. The findings are valuable for future clinical diagnosis and customized rehabilitation.

This research finds differences in OFC activation among addicts who take different types of drugs, and arrives at the conclusion that the right OFC activation of methamphetamine abusers is the highest, followed by that of mixed drug abusers and heroin abusers. The conclusion with left OFC activation is the same.
Existing research mostly focuses on moods, cognition and attention when studying drug rehabilitation. This paper introduces the fNIRS device to obtain the physiological data, and performed intelligent algorithm analysis and statistical analysis. The findings are consistent with existing conclusions that drugs have impact on brain functions. This research is innovative in that it makes classified study on the addicts who use different types of drugs. In theory, this paper enriches the empirical discussion on the impact of different types of drugs on human brains; and in practice, it provides guidance on customized drug rehabilitation.

\section{Abbreviations}

fNIRS: functional near-infrared spectroscopy; LDA: Linear discriminant analysis; SVM: Support vector machine; CNN: Convolutional Neural Network;

PFC: Prefrontal Cortex; FPC: Frontpolar prefrontal cortex; OFC: Orbitofrontal Cortex; VLPFC: Ventrolateral prefrontal Cortex; DLPFC: Dorsolateral prefrontal cortex; EEG: electroencephalography; HbO2: oxyhemoglobin;

Hbb: deoxyhemoglobin; MBLL: Modified lambert-beer law; NIRS: NIRSIT; DSM-

5: Diagnostic and Statistical Manual of Mental Disorders Five Edition;

LSD: lysergic acid diethylamide; MDMA: 3,4-

Methylenedioxymethamphetamine; $\mathrm{N}_{2} \mathrm{O}$ : Nitrous oxide; PFC: Prefrontal cortex; iRISA: impaired response inhibition and salience attribution

\section{Acknowledgements}

The authors thank the Shanghai Drug Rehabilitation Administration Bureau and Shanghai Qingdong Drug Rehabilitation Center for their support, and all the people who contribute to the writing of the article.

\section{Declaration of interest statement}

The authors declare that they have no known competing financial interests or personal relationships that could have appeared to influence the work reported in this paper.

\section{Authors' contributions}

$Y B, G S, Y L$ and $W W$ Provide paper ideas and experimental paradigm ideas design. GX Carry out the overall design and writing of the paper. XD Provided drug addicts as experimental data collection. All authors read and approved the final manuscript.

\section{Funding}

This project was supported by Key Research \& Development Project of National Science and Technique Ministry of China (No. 2018YFC0807405, No. 2018YFC1312903), National Natural Science Foundation of China (No. 
61976133), Defense Industrial Technology Development Program (JCKY2019413D002).

\section{Availability of data and materials}

All data used in this article are from Qingdong Drug Rehabilitation Center, Shanghai, China. The data is currently in a confidentiality agreement and cannot be disclosed.

\section{Declarations}

\section{Ethics approval and consent to participate}

The study was conducted in accordance with the declaration of Helsinki and was approved by the Ethics Committee of Shanghai University (Approval Number. ECSHU2020-071). Informed consent was obtained from all individual participants include in the study.

\section{Consent for publication}

Not applicable

\section{Competing interests}

The authors declare that they have no competing interests.

\section{Author details}

${ }^{1}$ School of Mechanical and Electrical Engineering and Automation, Shanghai University, Shanghai 200444, China. ${ }^{2}$ Department of Radiology \& Functional and Molecular Imaging Key Lab of Shaanxi Province, Tangdu Hospital, Fourth Military Medical University, Xi'an 710038, Shaanxi, China. ${ }^{3}$ Shanghai Drug Rehabilitation Administration Bureau, Shanghai 200080, China.

Received: 1 August 2021 Accepted: 11 November 2021 Published online: 25 November 2021

\section{References}

1. Jobsis FF. Noninvasive infrared monitoring of cerebral and myocardial oxygen sufficiency and circulatory parameters. Science. 1977;198(4323): 1264-7. https://doi.org/10.1126/science.929199.

2. Lancia S, Choi J, Baek J, Mammarella S, Bianco D, Quaresima V, et al. Trail making test induces prefrontal cortex activation as revealed by a cw wearable-wireless fNIRS/DOT imager. Adv Exp Med Biol. 2018:139-44. https://doi.org/10.1007/978-3-319-91287-5_22.

3. Huhn AS, Brooner RK, Sweeney MM, Yip SW, Ayaz H, Dunn KE. Increased neural activity in the right dorsolateral prefrontal cortex during a risky decision-making task is associated with cocaine use in methadonemaintained patients. Drug Alcohol Depen. 2019;205:107650. https://doi. org/10.1016/j.drugalcdep.2019.107650.

4. Ferrari $\mathrm{M}$, Quaresima V. A brief review on the history of human functional near infrared spectroscopy (fNIRS) development and fields of application. Neuroimage. 2012;63(2):921-35. https://doi.org/10.1016/j.neuroimage.2012. 03.049.

5. Kroczek AM, Haeussinger FB, Fallgatter AJ, Batra A, Ehlis AC. Prefrontal functional connectivity measured with near-infrared spectroscopy during smoking cue exposure. Addict Biol. 2015;22(2):513-22. https://doi.org/1 $0.1111 / a d b .12344$

6. Liu T, Liu X, Yi L, Zhu C, Markey PS, Pelowski M. Assessing autism at its social and developmental roots: A review of Autism Spectrum Disorder studies using functional near-infrared spectroscopy. Neurolmage. 2017;185: 955-67. https://doi.org/10.1016/j.neuroimage.2017.09.044

7. Dresler T, Schecklmann M, Ernst LH, Pohla C, Warrings B, Fischer M, et al. Recovery of cortical functioning in abstinent alcohol-dependent patients: Prefrontal brain oxygenation during verbal fluency at different phases during withdrawal. World J Biol Psychia. 2012;13(2):135-45. https://doi.org/1 0.3109/15622975.2011.564654.

8. Okada N, Takahashi K, Nishimura Y, Koike S, Ishii-Takahashi A, Sakakibara E, et al. Characterizing prefrontal cortical activity during inhibition task in methamphetamine-associated psychosis versus schizophrenia: a multichannel near-infrared spectroscopy study. Addict Biol. 2016;21(2):489-03. https://doi.org/10.1111/adb.12224.

9. Yamamuro K, Kimoto S, lida J, Kishimoto N, Nakanishi Y, Tanaka S, et al. Reduced Prefrontal Cortex Hemodynamic Response in Adults with Methamphetamine Induced Psychosis: Relevance for Impulsivity. PLoS One. 2016;11(4):e0152373. https://doi.org/10.1371/journal.pone.0152373.
10. Ceceli AO, Bradberry CW, Goldstein RZ. The neurobiology of drug addiction: cross-species insights into the dysfunction and recovery of the prefrontal cortex. Neuropsychopharmacol. 2021. https://doi.org/10.1038/s41386-02101153-9.

11. Dieler AC, Tupak SV, Fallgatter AJ. Functional near-infrared spectroscopy for the assessment of speech related tasks. Brain Lang. 2012;121:90-109. https:// doi.org/10.1016/j.bandl.2011.03.005

12. Etkin $A$, Egner $T$, Kalisch $R$. Emotional processing in anterior cingulate and medial prefrontal cortex. Trends Cogn Sci. 2011;15(2):85-3. https://doi.org/1 0.1016/j.tics.2010.11.004.

13. Alegria AA, Wulff M, Brinson $H$, Barker GJ, Norman LJ, et al. Real-time fMRI neurofeedback in adolescents with attention deficit hyperactivity disorder. Hum Brain Mapp. 2017;38(6):3190-209. https://doi.org/10.1002/hbm.23584

14. Linden DE. Neurofeedback and networks of depression. Dialogues Clin Neuro. 2014;16(1):103-12. https://doi.org/10.31887/DCNS.2014.16.1/dlinden.

15. Hosseini SMH, Pritchard-Berman M, Sosa N, Ceja A, Kesler SR. Task-based neurofeedback training: A novel approach toward training executive functions. Neurolmage. 2016;134:153-9. https://doi.org/10.1016/j.neuroima ge.2016.03.035.

16. Hofmann MJ, Dambacher M, Jacobs AM, Kliegl R, Radach R, Kuchinke L. Occipital and orbitofrontal hemodynamics during naturally paced reading An fNIRS study. Neurolmage. 2014;94:193-202. https://doi.org/10.1016/j. neuroimage.2014.03.014

17. Kim JE, Kim GH, Hwang J, Kim JY, Renshaw PF, Yurgelun-Todd DA, et al. Metabolic alterations in the anterior cingulate cortex and related cognitive deficits in late adolescent methamphetamine users. Addict Biol. 2016;23(1): 327-6. https://doi.org/10.1111/adb.12473.

18. Lyoo IK, Yoon S, Kim TS, Lim SM, Choi Y, Kim JE, et al. Predisposition to and effects of methamphetamine use on the adolescent brain. Mol Psychiatr. 2015:20(12):1516-4. https://doi.org/10.1038/mp.2014.191.

19. McCarthy JJ, Leamon MH, Willits NH, Salo R. The Effect of Methadone Dose Regimen on Neonatal Abstinence Syndrome. J Addict Med. 2015:9(2):10510. https://doi.org/10.1097/ADM.0000000000000099.

20. May AC, Aupperle RL, Stewart JL. Dark Times: The Role of Negative Reinforcement in Methamphetamine Addiction. Front Psychiatry. 2020;11: 114. https://doi.org/10.3389/fpsyt.2020.00114.

21. Kohno M, Dennis L, McCready H, Schwartz DL, Hoffman WF, Korthuis PT. A preliminary randomized clinical trial of naltrexone reduces striatal resting state functional connectivity in people with methamphetamine use disorder. Drug Alcohol Depen. 2018;192:186-2. https://doi.org/10.1016/j. drugalcdep.2018.07.045.

22. Paulus M. Behavioral and functional neuroimaging evidence for prefrontal dysfunction in methamphetamine- dependent subjects. Neuropsychopharmacol. 2002;26(1):53-63. https://doi.org/10.1016/S0893-133 X(01)00334-7.

23. Courtney KE, Ghahremani DG, Ray LA. The effects of pharmacological opioid blockade on neural measures of drug Cue-reactivity in humans. Neuropsychopharmacol. 2016;41(12):2872-1. https://doi.org/10.1038/npp.201 6.99 .

24. Johnson EC, Tillman R, Aliev F, Meyers JL, Salvatore JE, Anokhin AP, et al Exploring the relationship between polygenic risk for cannabis use, peer cannabis use, and the longitudinal course of cannabis involvement. Addiction. 2018;114(4):687-97. https://doi.org/10.1111/add.14512.

25. Kim JM, Choi JK, Choi M, Ji M, Hwang G, Ko SB, et al. Assessment of cerebral autoregulation using continuous-wave near-infrared spectroscopy during squat-stand maneuvers in subjects with symptoms of orthostatic intolerance. Sci Rep. 2018;8(1):13257. https://doi.org/10.1038/s41598-018-31 $685-y$.

26. Shin J, Kwon J, Choi J, Im CH. Performance enhancement of a braincomputer interface using high-density multi-distance NIRS. Sci Rep. 2017; 7(1):16545. https://doi.org/10.1038/s41598-017-16639-0.

27. Kim KY, Oh JE. Evaluation of pharmaceutical abuse and illicit drug use in South Korea by wastewater-based epidemiology. J Hazard Mater. 2020;396: 122622. https://doi.org/10.1016/j.jhazmat.2020.122622.

28. Strickland JC, Havens JR, Stoops WW. A nationally representative analysis of "twin epidemics": Rising rates of methamphetamine use among persons who use opioids. Drug Alcohol Depen. 2019;204:107592. https://doi.org/10.1 016/j.drugalcdep.2019.107592.

29. Huang S, Dai Y, Zhang C, Yang C, Huang Q, Hao W, et al. Higher impulsivity and lower grey matter volume in the bilateral prefrontal cortex in long-term abstinent individuals with severe methamphetamine use disorder. Drug 
Alcohol Depen. 2020;212:108040. https://doi.org/10.1016/j.drugalcdep.2020.1 08040.

30. Xu X, Huang E, Luo B, Cai D, Zhao X, Luo Q, et al. Methamphetamine exposure triggers apoptosis and autophagy in neuronal cells by activating the C/EBPß-related signaling pathway. Faseb J. 2018;32(12):6737-9. https:// doi.org/10.1096/fj.201701460RRR.

31. Ma T, Sun Y, Ku Y. Effects of Non-invasive Brain Stimulation on Stimulant Craving in Users of Cocaine, Amphetamine, or Methamphetamine: A Systematic Review and Meta-Analysis. Front Neurosci. 2019;13:1095. https:// doi.org/10.3389/fnins.2019.01095.

32. Lefrancois E, Belackova V, Silins E, Latimer J, Jauncey M, Shimmon R, et al. Substances injected at the Sydney Supervised Injecting Facility: A chemical analysis of used injecting equipment and comparison with self-reported drug type. Drug Alcohol Depen. 2020;209:107909. https://doi.org/10.1016/j. drugalcdep.2020.107909.

33. Kruyer A, Scofield MD, Wood D, Reissner KJ, Kalivas PW. Heroin Cue-Evoked Astrocytic Structural Plasticity at Nucleus Accumbens Synapses Inhibits Heroin Seeking. Biol Psychiatry. 2019;86(11):811-9. https://doi.org/10.1016/j. biopsych.2019.06.026.

34. Chen C, Florian K, Rajesh K, Max R, Christian K, Florian S, et al. Recovering the superficial microvascular pattern via diffuse reflection imaging: phantom validation. Biomed Eng Online. 2015;14:87. https://doi.org/10.1186/s12938-01 5-0081-x.

35. Ricci RW, Ditzler M, Nestor LP. Discovering the Beer-Lambert Law. J Chem Educ. 1994;71(11):983-5. https://doi.org/10.1021/ed071p983.

36. Uludag K, Kohl M, Steinbrink J, Obrig H, Villringer A. Cross talk in the Lambert-Beer calculation for near-infrared wavelengths estimated by Monte Carlo simulations. J Biomed Opt. 2002;7(1):51. https://doi.org/10.1117/1.142 7048.

37. Baker WB, Parthasarathy AB, Busch DR, Mesquita RC, Greenberg JH, Yodh AG. Modified Beer-Lambert law for blood flow. Biomed Opt Express. 2014; 5(11):4053. https://doi.org/10.1364/BOE.5.004053.

38. Leung TS, Elwell CE, Delpy DT. Estimation of cerebral oxy- and deoxyhaemoglobin concentration changes in a layered adult head model using near-infrared spectroscopy and multivariate statistical analysis. Phys Med Bio. 2005;50(24):5783-98. https://doi.org/10.1088/0031-9155/ 50/24/002.

39. Patterson MS, Wilson BC, Wyman DR. The propagation of optical radiation in tissue I. Models of radiation transport and their application. Laser Med Sci. 1991;6(2):155-8. https://doi.org/10.1007/BF02032543.

40. Delpy DT, Cope M, Zee P, Arridge S, Wray S, Wyatt J. Estimation of optical pathlength through tissue from direct time of flight measurement. Phys Med Bio. 1988;33(12):1433-42. https://doi.org/10.1088/0031-9155/33/12/008.

41. Franceschini MA, Boas DA. Noninvasive measurement of neuronal activity with near-infrared optical imaging. Neurolmage. 2004;21(1):372-86. https:// doi.org/10.1016/j.neuroimage.2003.09.040.

42. van der Zee P, Cope M, Arridge SR, Essenpreis M, Potter LA, Edwards AD, et al. Experimentally measured optical pathlengths for the adult head, calf and forearm and the head of the newborn infant as a function of inter optode spacing. Adv Exp Med Biol. 1992;316:143-53. https://doi.org/10.1 007/978-1-4615-3404-4_17.

43. Essenpreis M, Elwell CE, Cope M, van der Zee P, Arridge SR, Delpy DT. Spectral dependence of temporal point spread functions in human tissues. Appl Opt. 1993;3(4):418-25. https://doi.org/10.1364/AO.32.000418.

44. Duncan A, Meek JH, Clemence M, Elwell CE, Fallon P, Tyszczuk L, et al. Measurement of cranial optical pathlength as a function of age using phase resolved near-infared spectroscopy. Pediatr Res. 1996;39(5):889-94. https:// doi.org/10.1203/00006450-199605000-00025.

45. Cope M, Maniewski R, Kozluk E, Zbiec A, Nosek A, Walczak F. Near-infrared spectroscopy in evaluation of cerebral oxygenation during vasovagal syncope. Physiol Meas. 2004;25(4):823-36. https://doi.org/10.1088/ 0967-3334/25/4/004

46. Brekke PH, Rama T, Pilan I, Nytro O, Ovrelid L. Synthetic data for annotation and extraction of family history information from clinical text. J Biomed Semant. 2021;12(1):11. https://doi.org/10.1186/s13326-021-00244-2.

47. Jin XH, Zhao MB, Chow TWS, Pecht M. Motor Bearing Fault Diagnosis Using Trace Ratio Linear Discriminant Analysis. IEEE Trans Ind Electron. 2014;61(5): 2441-51. https://doi.org/10.1109/TIE.2013.2273471.

48. Bi L, Feng DD, Fulham M, Kim J. Multi-Label classification of multi-modality skin lesion via hyper-connected convolutional neural network. Pattern Recognit. 2020;107:107502. https://doi.org/10.1016/j.patcog.2020.107502.
49. Casteleiro MA, Demetriou G, Read W, Prieto MJF, Maroto N, Fernandez DM, et al. Deep learning meets ontologies: experiments to anchor the cardiovascular disease ontology in the biomedical literature. J Biomed Semant. 2018;9(1):13. https://doi.org/10.1186/s13326-018-0181-1.

50. Feng R, Liu X, Chen J, Chen DZ, Gao H, Wu J. A deep learning approach for colonoscopy pathology WSI analysis: accurate segmentation and classification. IEEE J Biomed Health. 2020. https://doi.org/10.1109/JBHI.2020.3 040269.

51. Holderness E, Miller N, Cawkwell P, Bolton K, Meteer M, Pustejovsky J, et al. Analysis of risk factor domains in psychosis patient health records. J Biomed Semant. 2019;10(1):19. https://doi.org/10.18653/v1/W18-5615.

52. Baler RD, Volkow ND. Drug addiction: the neurobiology of disrupted selfcontrol. Trends Mol Med. 2006;12(12):559-66. https://doi.org/10.1016/j. molmed.2006.10.005

53. Volkow ND, Fowler JS, Wang GJ. The addicted human brain: insights from imaging studies. J Clin Invest. 2003;111(10):1444-51. https://doi.org/10.1172/ JCl18533.

54. Ning M, Ying L, Nan L, et al. Addiction related alteration in resting-state brain connectivity. Neuroimage. 2010;49(1):738-44. https://doi.org/10.1016/j. neuroimage.2009.08.037.

55. Bechara A. Decision making, impulse control and loss of willpower to resist drugs: a neurocognitive perspective. Nat Neurosci. 2005;8(11):1458. https:// doi.org/10.1038/nn1584.

56. Wiers RW, Bartholow BD, van den Wildenberg E, Thush C, Engels RC, et al. Automatic and controlled processes and the development of addictive behaviors in adolescents: a review and a model. Pharmacol Biochem Be. 2006;86(2):263-83. https://doi.org/10.1016/j.pbb.2006.09.021.

57. Lee TMC, Zhou WH, Luo XJ, Yuen KS, Ruan XZ, Weng XC. Neural activity associated with cognitive regulation in heroin users: A fMRI study. Neurosci Lett. 2005;382:211-6. https://doi.org/10.1016/.jneulet.2005.03.053.

58. Maeyer J, Vanderplasschen W, Broekaert E. Quality of life among opiatedependent individuals: a review of the literature. Int J Drug Policy. 2010; 21(5):364-80. https://doi.org/10.1016/j.drugpo.2010.01.010.

59. Baldacchino A, Balfour DJK, Passetti F, Humphris G, Matthews K. Neuropsychological consequences of chronic opioid use: A quantitative review and meta-analysis. Neurosci Biobehav Rev. 2012;36(9):2056-68. https://doi.org/10.1016/.neubiorev.2012.06.006.

60. Fattore L, Diana M. Drug addiction: an affective-cognitive disorder in need of a cure. Neurosci Biobehav Rev. 2016;65:341-61. https://doi.org/10.1016/j. neubiorev.2016.04.006

\section{Publisher's Note}

Springer Nature remains neutral with regard to jurisdictional claims in published maps and institutional affiliations.
Ready to submit your research? Choose BMC and benefit from:
- fast, convenient online submission
- thorough peer review by experienced researchers in your field
- rapid publication on acceptance
- support for research data, including large and complex data types
- gold Open Access which fosters wider collaboration and increased citations
- maximum visibility for your research: over $100 \mathrm{M}$ website views per year
At BMC, research is always in progress.
Learn more biomedcentral.com/submissions 